\title{
Evaluation of the True Effect of Experimental Parameters on the Reduction / Oxidation Processes Observed in Fast-Atom Bombardment/Liquid Secondary Ion Mass Spectrometry
}

\author{
J. Visentini, D. Zidarov, M. Allard, and M. J. Bertrand \\ Regional Center for Mass Spectrometry, Department of Chemistry, University of Montreal, Quebec, Canada
}

\begin{abstract}
The peak intensities observed in the molecular ion regions of fast-atom bombardment/liquid secondary ion mass spectra contain contributions from the parent ion species, its one- and two-electron reduction/oxidation products, and chemical background signal due to beaminduced damage. There are several solution and instrumental parameters that can affect the distribution of peak intensities in the molecular ion region. In this study, the analyte concentration and primary beam density and energy were varied systematically to investigate their effects on the measured peak intensities. A computer algorithm, Simbroc (Simulated Background and Reduction/Oxidation Calculations), was designed to deconvolute the observed intensities into their individual components so that the true effects of experimental parameters on redox extent and background levels could be evaluated. The algorithm is based on a comprehensive seven-variable mathematical model for experimental data simulation. The results obtained using the algorithm after its validation indicate that the primary beam energy does not significantly affect redox extent or background levels. Changes in analyte concentration and primary beam density tend to play a more important role in the generation of redox products and beam-induced damage. The background level generally increases as the analyte concentration is lowered for the peptide systems used in this study. An increase in the primary beam density often leads to higher background levels, although the effect is less detectable for samples that have a low (less than $3 \%$ ) background signal. The apparent two-electron reduction is generally lower at the higher concentrations; however, the "true" reduction occurring for pentaphenylalanine does not show a significant concentration effect. (J Am Soc Mass Spectrom 1993, 4, 482-492)
\end{abstract}

$\mathrm{T}$ The reduction reactions often observed during fast atom bombardment/liquid secondary ion mass spectrometry (FAB/LSIMS) analysis have attracted considerable interest since they were first reported to occur in glycerol solutions of various inorganic and organic solutes [1]. Later reports showed that reduction processes can also be observed in different classes of compounds, including organometallic species, dyes, and biomolecules, such as peptides, nucleosides, and quinones [1-28]. The reduction products formed during the $\mathrm{FAB}$ /LSIMS process manifest themselves experimentally as $\mathrm{A}+n\left(\mathrm{~A}=[\mathrm{M}+\mathrm{H}]^{+} ; n \geq 1\right)$ peak intensities that are larger than would be expected

Address reprint requests to Michel J. Bertrand, Regional Center for Mass Spectrometry, Department of Chemistry, University of Montreal, P.O. Box 6128, Station A, Montreal, Qucbec, Canada H3C 3J7. on the basis of natural isotopic abundances. The extent of the reduction observed has been shown to be dependent both on solution and on instrumental parameters. Because different analyte/matrix combinations will show a different extent of reduction, the analyte structure and matrix composition are important factors to consider when studying reduction reactions [28]. Besides the functional groups present in the analyte and matrix molecules, the concentration of analyte in the matrix has also been noted to affect the positive deviation of $(A+1) / A$ and $(A+2) / A$ isotopic ratios from theoretical values. Reports on the concentration effect indicate that the measured isotopic peak intensities will increase as the solution concentration is decreased $[7,13,15,28)$.

Instrumental parameters associated with the FAB/LSIMS source conditions may also play an im- 
portant role in determining the extent of reduction observed. Those parameters that have been studied include time of irradiation $[8,14,16,17,23]$; primary beam energy $[15,20,28]$; and bombarding beam density (flux) $[12,15,20,23,28]$. Wirth et al. [8] observed a time dependence in their $F A B$ analysis $\left(\mathrm{Xe}^{\circ}\right.$ primary beam) of azo-group-containing peptides. This time dependence, however, was not observed when the primary beam was composed of $\mathrm{Cs}^{+}$ions, even though the current density was kept the same as that of the xenon beam. Results obtained in this laboratory using different peptide samples and a $\mathrm{Cs}^{+}$primary ion beam generally indicate no significant increases in reduction with time [29]. The primary beam energy and density have also been investigated. Kazakoff et al. [15] noted that the concentration effect for the $(A+1) / A$ ratio of a pyridinium salt was lost when the primary beam energy was decreased from 9 to $5 \mathrm{keV}$. Our LSIMS work conducted with different peptides showed a concentration effect regardless of the primary beam energy used [28]. Reynolds and Cook [20] studied the effects of primary beam energy and density on the reduction of methylene blue. They noted that the extent of reduction is lowered by raising the primary beam energy but that the effect of varying the energy of bombardment is proportionately smaller than that of changing the primary beam density. In fact, in their work with methylene blue, the energy effect on the enhancement factor becomes much less important at primary beam energies above $5 \mathrm{keV}$. The primary beam density, however, plays a more important role in determining the extent of reduction. An increase in the beam density caused substantial increases in the enhancement ratios measured for methylene blue $[20,30]$ and for different peptide systems [28]. For systems in which the extent of reduction is large, therefore, it is more important to lower the primary beam density than it is to raise the primary beam energy [20].

Several contributions, including natural isotopic abundances, background signal (due to beam-induced chemical damage of analy le, matrix, and cuntbined analyte/matrix species), and reduced/oxidized species are usually present in the molecular ion region, and they are not easy to isolate. The methods currently used to express the extent of reduction, commonly referred to as "enhancement factors," assign the excess signal in the $\mathrm{A}+n$ peaks entirely to reduction products $[15,20]$. This approach, however, measures the "apparent" reduction rather than the "true" reduction extent by overlooking contributions of beam-induced background signal and isotopic carryover from other processes affecting peak intensities in the molecular ion region. Furthermore, it is very difficult to make interlaboratory comparisons because parameters affecting the reduction processes are sometimes omitted in the literature.

Methods for evaluating and removing background signal from conventional FAB/LSI mass spectra have been developed for peptide sequencing programs
[31-34]. This filtering of beam-induced background peaks from the mass spectra is essential in the isolation of sequence ions from the peak-at-every-mass distribution commonly observed in FAB/LSI spectra. It is surprising, therefore, that the problem of background interference, which can affect the apparent redox processes in the molecular ion region, had not been addressed until recently [20-22, 28]. To determine quantitatively the contribution of redox processes to the intensity distribution of peaks in the parent ion cluster, the natural isotopic abundances and the background signal must be deconvoluted from the overall peak intensities measured in this mass region. Deconvolution procedures that have been presented in the literature $[4,18]$ are performed by solving a determined set of linear equations and then calculating a least-squares fit. Both of these procedures, however, are vulnerable to errors due to chemical interference because a determined set of equations has only one solution that may be mathematically correct yet physically insignificant. Furthermore, the background contribution, which may be important, is neglected in these methods. In more recent work. Vekey [21] suggested that the background level be subtracted directly from the peak intensities in the molecular ion region and that a "monoisotopic" spectrum be determined manually. This "monoisotopic" spectrum, which represents only the lowest mass isotopes of the species with various numbers of hydrogen atoms, would give a true representation of the distribution of oxidation/reduction products relative to the parent ion. The background value to be subtracted from observed isotopic peak intensities is assumed to be constant, and its magnitude is determined by the mean intensity of the smallest peaks beforc and after the cluster to be studied. This direct subtraction method, however, which can be rather subjective in terms of the peaks used, can produce errors in the evaluation of background level and reduction/oxidation extent because the background is not always uniformly distributed in the molecular ion region [22]. By fixing the background at a constant value, this approach therefore offers no flexibility in the optimization of the variables representing the redox extent. Furthermore, the background pattern may change with bombardment time, analyte concentration, primary beam flux, analyte structure, and matrix composition, thereby further propagating errors.

To determine the "true" extent of reduction processes, a simple mathematical model that simulates experimental data was recently developed in our laboratory [28]. Because two-electron reduction processes were often predominant for the peptide samples of interest, this earlier work considered only the contributions of natural isotopic abundances, two-electron reduction products, and background signal to the peak intensity distribution in the molecular ion cluster. Although this model was sensitive to the interference of other redox processes, it was unable to account for them when they occurred to a significant extent and 
simultaneously with the two-electron reduction process. It was clear from the results of this earlier work that a more general method that could reliably deconvolute the ion intensities for a wider range of chemical systems was needed. This task was achieved by using a more comprehensive seven-variable model on which the computer program Simbroc (Simulated Background and Reduction-Oxidation Calculations) described in this work is based. The results from this program can be used to study the variations of the trends in "true" rather than "apparent" reduction and oxidation processes under various experimental conditions.

\section{Experimental}

The peptides (pentaphenylalanine and bradykinin) and the methylene blue dye were purchased from Sigma Chemical Co. (St. Louis, MO); pressinoic acid was obtained from Bachem Inc. (Torrance, CA); and deaminoarginine vasopressin (DDAVP-ACOH) was provided by BioMega Inc. (Laval, Canada). The acetal (4-decyl-2-phenyl-1,3-dioxolane) was synthesized through a condensation reaction between benzaldehyde and 1,3-dodecanol. Glycerol (Aldrich Chemiral Co., Milwaukee, WI) was used to prepare the solutions used in this study. Two-microliter aliquots of the solutions, ranging in concentration from 0.005 to $0.08 \mathrm{M}$, were deposited on the probe tip using a glass syringe. All mass spectral data were acquired using a VG AutospecQ hybrid mass spectrometer (Manchester, UK) equipped with a standard VG LSIMS ion source using a variable energy (0-50 keV) cesium ion gun. The instrument was opcrated at an accelerating voltage of $8 \mathrm{kV}$ and a mass resolution of 2000 (10\% valley definition). Accurate isotopic peak intensity measurements were made using the narrow-voltage-scanning technique, and data were accumulated using the multichannel acquisition mode at 50-s scan intervals. The signals at the different nominal masses present in the parent molecular ion cluster were integrated, and peak areas were used for the determination of accurate isotopic peak ratios. Data were averaged over a 5-min period to maximize reproducibility, which was on the order of $10 \%$. The procedure was repeated at least three times. All data acquisition and treatment were performed using the VG Opus software (Version 1.6F) and the programs Srbc (Simulated Reduction and Background Calculations) [28] and Simbroc developed in this laboratory. The program Simbroc, written in Fortran 77, has a storage size of 150 kbytes. Calculations can be performed on an IBM-compatible computer or on a VAX Station (Digital Equipment Corp., Maynard, MA). Because a minimum random-access memory of 1 Mbyte is needed to ensure a reasonable processing time, it is recommended that this program be executed on a PC 386 with a mathematical coprocessor or a PC 486 series computer. The execution times on these types of systems using a 5.0 disk-oper- ating system will be on the order of $15 \mathrm{~s}$. Simbroc may also be operated using either a VAX 2000 or VAX 3000 computer equipped with a VMS operating system (version 5.4 or earlier). The execution times for these systems will be on the order of 10-15 $\mathrm{s}$ for the VAX 2000 and approximately $5 \mathrm{~s}$ for the VAX 3000 . Further information about this program is available on request. The beam density values cited in this work were obtained by using a Faraday cup detector placed at the target, as described earlier [28]. This method involves measuring the current reaching the LSIMS probe tip using a Keithley 485 autoranging picoammeter and then dividing this reading by the probe tip surface to provide a beam density value.

\section{Results and Discussion}

Oxidation/reduction processes have been observed in different chemical systems analyzed under FAB / LSIMS conditions [1-28, 35-38]. These processes can affect the distribution of peak intensities in the molecular ion region and thereby interfere with the determination of accurate isotopic peak measurements. Because empirical formula determination is often dependent on the accuracy of isotopic peak measurements [31-32, 39], it is important to evaluate the contributions of the oxidation/reduction products to the overall peak intensities measured. A typical molecular ion cluster profile acquired using narrow-voltage scanning in the multichannel acquisition mode for a pentaphenylalanine sample $(0.08 \mathrm{M}, 14 \mathrm{keV}, 0.018$ $\mu \mathrm{A} / \mathrm{mm}^{2}$ ) is shown in Figure 1 . The symbols $I_{k}$ represent the peak intensities observed experimentally, with the subscript $k$ denoting the position of the peak in the molecular ion cluster relative to the parent ion that occurs at $k=0$. The peak intensities $I_{k}$ measured experimentally contain contributions from natural isotopic abundances, chemical background signal, and

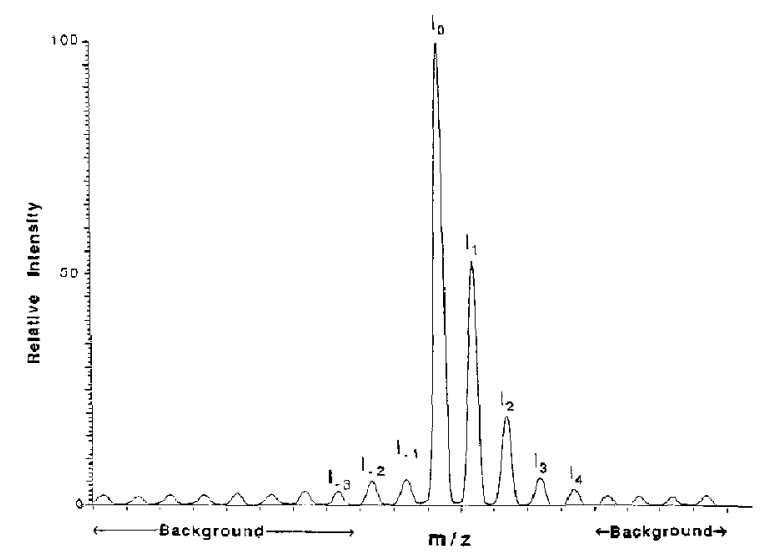

Figure 1. Typical narrow-voltage-scanning spectrum showing the nomenclature used to identify observed peak intensities in the molecular ion cluster region. 
oxidized/reduced species. Although the exact reduction/oxidation mechanisms are not yet known, it is accepted that only those redox processes that result in hydrogen additions or losses will affect the isotopic peak intensities in the molecular ion region. For cases in which the analyte is neutral prior to the $\mathrm{FAB}$ process, therefore, we define the reduction/oxidation processes as shown in eqs $1-5$.

$$
\begin{aligned}
& \mathrm{Ox}_{2}: \mathbf{M} \stackrel{-2 \mathrm{H}^{+}}{\longrightarrow}[\mathrm{M}-2 \mathrm{H}] \stackrel{+\mathrm{H}^{+}}{\longrightarrow}[\mathrm{M}-\mathrm{H}]^{+} \\
& \mathrm{Ox}_{1}: \mathbf{M} \stackrel{-\mathrm{H}^{+}}{\longrightarrow}[\mathrm{M}-\mathrm{H}]^{-} \stackrel{+\mathrm{H}^{+}}{\rightarrow} \mathbf{M}^{+} \\
& \operatorname{Red}_{0}: \mathbf{M} \stackrel{+\mathrm{H}^{+}}{\longrightarrow}[\mathrm{M}+\mathrm{H}]^{+} \\
& \operatorname{Red}_{1}: \mathbf{M} \stackrel{+\mathrm{H}^{+}}{\longrightarrow}[\mathrm{M}+\mathrm{H}]^{*} \stackrel{+\mathrm{H}^{\prime}}{\longrightarrow}[\mathrm{M}+2 \mathrm{H}]^{+} \\
& \operatorname{Red}_{2}: \mathbf{M} \stackrel{+2 \mathrm{H}^{+}}{\longrightarrow}[\mathrm{M}+2 \mathrm{H}] \stackrel{+\mathrm{H}^{+}}{\longrightarrow}[\mathbf{M}+3 \mathrm{H}]^{+}
\end{aligned}
$$

Where $Q x$ and Red designate those oxidation and reduction processes, respectively, that are accompanied by hydrogen transfers, and their subscripts denote the number of hydrogens/electrons transferred in the process. According to this nomenclature, therefore, the symbol $\operatorname{Red}_{0}$ refers to the signal due to the neutral $M$ species that is observed experimentally as the [M + $\mathrm{H}]^{+}$ion or the $\mathrm{M}^{+}$species in cases where the ion is charged prior to analysis. For the peptides used in this study, the $\operatorname{Red}_{0}$ variable denotes the parent $[M+H]^{+}$ species that has undergone neither reduction nor oxidation. It should be noted that eqs 1-5 show the products of the redox reactions and do not necessarily reflect the mechanisms involved. For example, in addition to the process described by eq 2, the one-electron oxidation process could also occur through direct ionization of $\mathrm{M}$; that is, $\mathrm{M} \rightarrow \mathrm{M}^{+}$. $+\mathrm{e}^{-}$. Both reaction pathways, however, lead to the formation of the same species, $\mathrm{M}^{+}$., which occurs at the same peak position regardless of the mechanism involved in its formation. In a similar way, the reaction product corresponding to a two-electron oxidation process in eq 1 could mechanistically be generated by elimination of a hydride ion (II ${ }^{-}$). To simplify the definition of processes, therefore, the equations listed here are not meant to represent all possible reaction pathways leading to the formation of the different redox species.

For chemical systems in which the analyte is charged prior to FAB analysis, such as for the methylene blue dye sample, the redox products are shifted $1 \mathrm{u}$ lower than in the systems described earlier. The definition of redox processes for these types of systems can be described by eqs $6-10$ :

$$
\begin{aligned}
& \mathrm{Ox}_{2}: \quad \mathrm{M}^{+} \stackrel{-2 \mathrm{H}}{\longrightarrow}[\mathrm{M}-2 \mathrm{H}]^{+} \\
& \mathrm{Ox} ; \quad \mathrm{M}^{+} \stackrel{-\mathrm{H}^{*}}{\longrightarrow}[\mathrm{M}-\mathrm{H}]^{+} \text {. } \\
& \operatorname{Red}_{0}: \mathrm{M}^{+} \rightarrow \mathbf{M}^{+} \\
& \operatorname{Red}_{1}: \mathbf{M}^{+} \stackrel{+\mathrm{H}^{*}}{\longrightarrow}[\mathrm{M}+\mathrm{H}]^{+} \text {. } \\
& \operatorname{Red}_{2}: \mathbf{M}^{+} \stackrel{+2 \mathrm{H}^{\circ}}{\longrightarrow}[\mathrm{M}+2 \mathrm{H}]^{+}
\end{aligned}
$$

Besides the reduced/oxidized species and natural isotopic abundances, the peak intensities shown in Figure 1 also include a contribution from background signal due to beam-induced chemical damage. This background signal can be observed experimentally because the peaks neighboring the molecular ion cluster profile (indicated in Figure 1) can occur in three different pattern types (Figure 2). Although a constant background pattern is sometimes observed (Figure 2a), it is also important to consider those cases where the background is distributed in a "picket-fence" pattern (Figure $2 b$ ) or as a modulated signal (Figure 2c). The mathematical model of Simbroc, which is proposed for the evaluation of redox processes in FAB/LSIMS, accounts for these different background pattern types by using two rather than one background variable, as is described later.

a

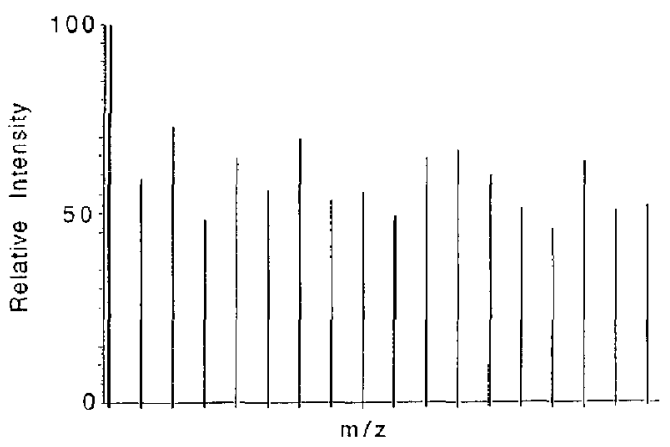

b
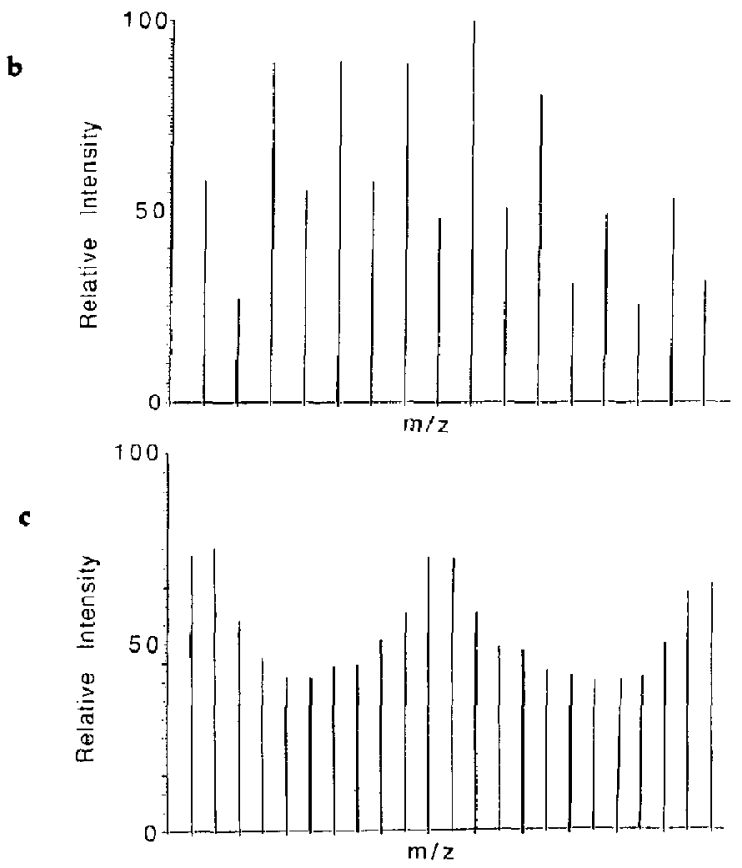

Figure 2. Three basic types of background distributions observed in FAB/LSI mass spectra: (a) constant, (b) "picket-fence," and (c) modulated signal. These background patterns were taken from experimental data. 
Earlier work conducted in this laboratory indicated that although a simple mathematical model considering only a constant background and two-electron reduction processes could be used to reveal trends in the "true" reduction processes occurring in FAB/LSIMS analysis of some peptides, an extended model was needed if the approach was to be of general use [28]. The contributions from one-electron reduction and from one- and two-electron oxidation products and those from a variable background signal were included in the development of the extended model. The system of eqs 11-18, shown below, is that on which the computer program Simbroc was based:

$$
\begin{aligned}
& I_{-3}=\text { Back }_{2} \\
& I_{-2}=O x_{2}+\text { Back }_{1} \\
& I_{-1}=\mathrm{A}_{1} * \mathrm{Ox}_{2}+\mathrm{Ox}_{1}+\mathrm{Back}_{2} \\
& I_{0}=\mathrm{A}_{2} * O x_{2}+\mathrm{A}_{1} * \mathrm{Ox}_{1}+\operatorname{Red}_{0} \\
& + \text { Back }_{1} \\
& I_{1}=\mathrm{A}_{3} * \mathrm{Ox}_{2}+\mathrm{A}_{2} * \mathrm{Ox}_{1}+\mathrm{A}_{1} * \operatorname{Red}_{0} \\
& +\operatorname{Red}_{1}+\text { Back }_{2} \\
& I_{2}=\mathrm{A}_{4} * \mathrm{Ox}_{2}+\mathrm{A}_{3} * \mathrm{Ox}_{1}+\mathrm{A}_{2} * \operatorname{Red}_{0} \\
& I_{3}=\begin{array}{l}
+A_{1} * \operatorname{Red}_{1}+\operatorname{Red}_{2}+\operatorname{Back}_{1} \\
\Lambda_{4} * \operatorname{Ox}_{1}+A_{3} * \operatorname{Rcd}_{0}+\Lambda_{2} * \operatorname{Red}_{1} \\
+A_{1} * \operatorname{Red}_{2}+\operatorname{Back}_{2}
\end{array} \\
& I_{1}=A_{1} * \operatorname{Red}_{0}+A_{3} * \operatorname{Red}_{1}+A_{2} * \operatorname{Red}_{2}
\end{aligned}
$$

Where $A_{1}, A_{2}, A_{3}$, and $A_{4}$ represent the first, second, third, and fourth isotopic contributions, respectively, and the variables Back 1 and Back 2 represent the level of background present in the mulecular ion region. This background signal, as mentioned earlier, can be observed experimentally as three different pattern types (Figure 2). The use of only one background variable would impose a constant background over the molecular ion region, thereby restricting the flexibility of the other variables. By using two variables, therefore, this model allows some flexibility in the evaluation of the background and redox product contributions to the overall peak intensities measured. Thus, if the background has a constant value over the mass range of the cluster, then Back ${ }_{1}$ and Back $_{2}$ will converge. If the background has a "picket-fence" distribution (Figure 2b), then Back ${ }_{1}$ and Back ${ }_{2}$ will take distinct and different values. Finally, if the background is a modulated signal, as in Figure $2 c$, then Back $_{1}$ and Back $_{2}$ will take two average values, thus minimizing the errors on background assessment and on the values of redox processes. The ion intensities measured in the molecular ion region can be simulated according to eqs 11-18, which include all of the variables of interest. It is the $I_{k}$ values, which include the contributions from background, natural isotopic abundances, and the oxidized/reduced species accordingly, that the program tries to best match with observed peak inten- sities. The model uses eqs $12-17$ to estimate values for the processes and eqs $11-18$ to optimize them.

'The algorithm flowchart for Simbroc is described in Figure 3. The input required for this program includes the empirical formula of the analyte and the experimental peak intensities for those peaks ranging from 3 $\mathrm{u}$ below $\left(I_{-3}\right)$ to $4 \mathrm{u}$ above $\left(I_{4}\right)$ the parent ion. The empirical formula is used to calculate the theoretical isotopic peak ratios $A_{1}, A_{2}, A_{3}$, and $A_{4}$. The program then proceeds to the simulation procedure, which relies on the Rosenbrock constrained optimization technique [40]. Because the Rosenbrock method is sensitive to the starting position (initial values) and to the step for each variable in the determination of a global minimum, the first step involves calculating an exact solution for eqs 12-17, where Back ${ }_{1}$ and Back ${ }_{2}$ values are set equal. This exact solution places the Rosenbrock technique at a reasonable starting point in the hyperspace. Once the starting values are set, then the background variables Back ${ }_{1}$ and Back $_{2}$ can become independent, and the solution set giving the best fit with experimental data can be found using the Rosenbrock optimization technique. Because the system of eqs

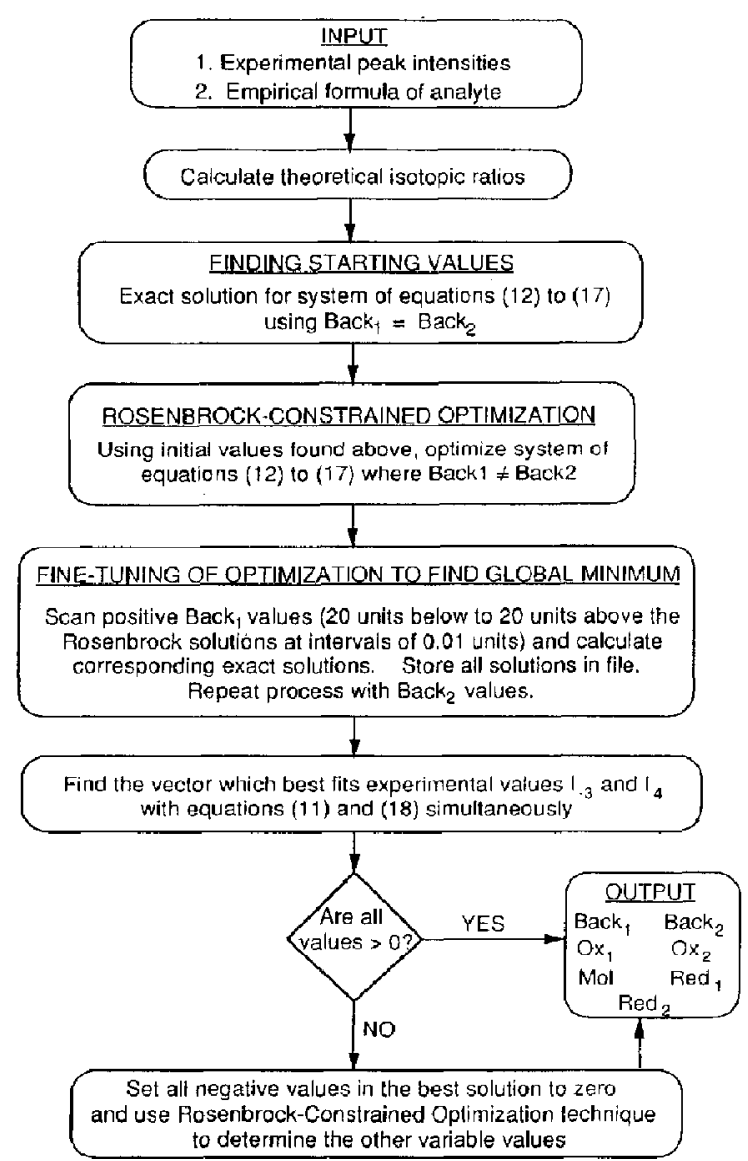

Figure 3. Flowchart of the algorithm used in Simbroc. 
$12-17$ is underdetermined, it has numerous solutions. Furthermore, because there may be several local minima in the global minimum region, the Rosenbrock technique is sometimes incapable of resolving the global minimum from all of the other solution sets in the neighboring region. A verification procedure for the Rosenbrock solution was therefore deemed necessary. The fine-tuming of the Rosenbrock optimization technique can be achieved using an external loop. That is, the Back 1 variable is scanned at all positive values from $20 \mathrm{u}$ below to $20 \mathrm{u}$ above the Rosenbrock solution and with an interval of $0.01 \mathrm{u}$. The exact solution sets corresponding to each Back $_{1}$ value are systematically stored in an array. This scanning process and storing of solution sets is then repeated with Back 2 values. The best fitting solution set in the array is determined by finding the vector that best matches eqs 11 and 18 simultaneously to experimental $I_{-3}$ and $I_{4}$ ion intensities, respectively. This procedure has the advantage that it does not force Back $_{2}$ to take the $I_{-3}$ value unless this value truly corresponds to $I_{-3}$. The flexibility afforded by this method should therefore minimize the errors encountered with the methods using a single background variable $[21,28]$. The global minimum is either confirmed or determined through the use of this scanning and best-fit procedure. Furthermore, this verification of the global minimum is also instrumental in finding a solution set that is physically significant. Because it is impossible to have a negative background level or redox process, should any value in the resultant vector be negative, the program will set these variables to zero and use the Rosenbrock method to readjust the values of the other variables. This adjustment of the solution vector is usually only needed in those cases where there are certain processes that are extreme. That is, the relative error involved in a process that occurs to an extent greater than approximatcly $70 \%$ may be large enough to cause the values of either nonexistent or only slight processes to result as negative values.

The validity of the results obtained using Simbroc, and the program's ability to handle the three types of background described earlier (Figure 2), were evaluated using lest examples in which the peak intensities were calculated for situations in which the background and redox variables were fixed at known values. These synthetic peak intensities were then entered into Simbroc $_{r}$ and the results obtained for examples assuming constant, "picket-fence," and modulated background signal are shown in Table 1 . In the cases of constant and "picket-fence" background distributions, Simbroc was able to find the correct values for each redox process, with at most $1 \%$ relative error. When the experimental background appears as a modulated signal, the relative error is larger owing to the lack of conformity between the distribution of three different background values involved in this pattern and the presence of only two background variables in the model. The results from the program indicate that the
Table 1. Ability of Simbroc to deal with the three basic types of background observed in FAB/LS mass spectra

\begin{tabular}{|c|c|c|c|c|c|c|}
\hline & \multicolumn{2}{|c|}{ Constant } & \multicolumn{2}{|c|}{ "Picket-fence" } & \multicolumn{2}{|c|}{ Modulated } \\
\hline & Actual & Simbroc & Actual & Simbroc & Actual & Simbroc \\
\hline Back, & 3 & 3.000 & 3 & 3.000 & 2.4 & 2.896 \\
\hline Back $_{2}$ & 3 & 3.004 & 5 & 5.004 & 3 & 3.170 \\
\hline Back $_{3}$ & - & - & - & - & 3.6 & - \\
\hline$O x_{2}$ & 5 & 5.000 & 5 & 5.000 & 5 & 4.830 \\
\hline$O x_{1}$ & 10 & 9.996 & 10 & 9.996 & 10 & 10.811 \\
\hline $\operatorname{Red}_{0}$ & 30 & 30.003 & 30 & 30.000 & 30 & 29.959 \\
\hline $\operatorname{Red}_{1}$ & 20 & 19.997 & 20 & 19.997 & 20 & 19.967 \\
\hline $\operatorname{Red}_{2}$ & 50 & 50.004 & 50 & 50.004 & 50 & 49.221 \\
\hline
\end{tabular}

model will average the background contribution and that the relative error involved in the redox processes is less than $10 \%$, which is on the order of the reproducibility of experimental data. In cases where other interference besides redox products and background signal contaminate the cluster, the impurity can be corrected if its identity is known. If the nature of the impurity is not known and is accidental in nature, the program will yield results that are not compatible with physical results. Although Simbroc cannot evaluate the redox extent and background levels under these conditions, it makes the user aware of contamination in the system by producing inconsistent results and a very poor match between simulated and experimental data. This type of situation has been observed in this laboratory for cases where intense matrix peaks or sample impurities interfered in the parent ion cluster region.

In cases where two-electron reduction occurs simultaneously with other redox processes, our earlier, more simple model could detect the presence of other redox processes occurring to an equal or greater extent than the two-electron reduction process but could not evaluate the processes. For example, one chemical system that our earlier program Srbc was unable to treat successfully was pressinoic acid in glycerol. The results obtained with Srbc and the more recent Simbroc for this disulfide bridge-containing peptide are shown in Table 2. The experimental conditions for this data set included a primary beam energy of $16 \mathrm{keV}$ and a primary beam density of $0.018 \mu \mathrm{A} / \mathrm{mm}^{\text {? }}$. The background values determined by Srbc, as shown in Table 2 , were far from those approximated experimentally from the $[\mathrm{M}+\mathrm{H}-4]-[\mathrm{M}+\mathrm{H}-8]$ peaks in the voltage scans (superscript d). It was evident from these poor matches that besides the two-electron reduction process, this chemical system was being affected by different reduction mechanisms. The same experimental data were later treated using Simbroc. The background values, Back 1 and Back $_{2}$, obtained using this method showed an absolute deviation on the order of 0.5-1\% from the observed intensities of those peaks surrounding the molecular ion cluster. In cases where the background is much smaller than the extent of redox processes, a larger relative error in the level of background can be expected. Nevertheless, an absolute 
Table 2. Concentration effect on the redox processes and background level for a pressinoic acid sample analyzed in glycerol $^{\text {a }}$

\begin{tabular}{|c|c|c|c|c|c|c|c|c|c|c|c|}
\hline \multirow{2}{*}{$\begin{array}{c}\text { Concentration } \\
\text { (M) }\end{array}$} & \multicolumn{8}{|c|}{ Simbroc $(\%)$} & \multicolumn{2}{|c|}{ Srbc $^{b} \%$} & \multirow{2}{*}{$\begin{array}{c}\text { Back }^{d} \\
\text { spectra \% }\end{array}$} \\
\hline & Back $_{1}$ & Back $_{2}$ & $\mathrm{Ox}_{2}$ & $O x_{1}$ & $\operatorname{Red}_{0}$ & $\operatorname{Red}_{1}$ & Red $_{2}$ & $\operatorname{Red}_{2}^{g}$ & Red & Back & \\
\hline 0.005 & 1.27 & 0.00 & 0.96 & 5.6 & 69.7 & 11.4 & 12.3 & 16.8 & 11.1 & 13.8 & 1.0 \\
\hline 0.025 & 0.00 & 0.00 & 0.99 & 2.7 & 86.8 & 3.6 & 5.9 & 6.7 & 5.7 & 4.6 & 0.5 \\
\hline 0.05 & 0.05 & 0.00 & 0.98 & 2.3 & 92.4 & 0.3 & 4.0 & 4.3 & 4.3 & 2.9 & 0.7 \\
\hline
\end{tabular}

error of $1 \%$ in the background would indicate only a small relative error in the substantially larger redox values calculated by Simbroc for such systems.

Examination of the redox values determined by Simbroc indicates that one-electron reduction is nearly as important as the two-electron reduction process for this system at the lower concentrations of 0.005 and $0.025 \mathrm{M}$. For the higher concentration of $0.05 \mathrm{M}$, the two-electron reduction process predominates. Because the extent of one-electron reduction was ignored by Srbc, the isotopic contributions of those species undergoing one-electron reduction were attributed to background, thereby generating erroneous background values and, consequently, erroneous two-electron reduction extent values. All background values and all results calculated using Srbc are given as a percentage of the base peak intensity in the molecular ion region. The redox extent values calculated by Simbroc, however, are normalized to give reduction/oxidation processes as percentages of the total processes observed (sum of $\mathrm{Ox}_{2}, \mathrm{Ox}_{1}, \operatorname{Red}_{0}, \operatorname{Red}_{1}$ and $\operatorname{Red}_{2}$ ). To compare the two-electron reduction extent calculated by both programs (Srbc and Simbroc), the values obtained using Simbroc were left unnormalized as percentages of the base peak intensity. Comparison of these values (superscript c, Table 2) with those obtained using Srbc indicates that the "true" extent of the two-electron reduction for pressinoic acid is higher than that calculated by Srbc. Clearly, Srbc had overevaluated the background contribution while sacrificing the extent of Iwo-electron reduction. This error was due to the absence of a variable in that model for one-electron reduction, which is important at the lower concentrations for this system. Simbroc results also indicate that the two-electron oxidation process is the least important process and that it does not change with analyte concentration. Also seen in Table 2, the quantity of parent ion species, $\operatorname{Red}_{0}$, increases with increasing concentration. It appears, therefore, that the oxidation/reduction processes are more likely to occur at lower concentrations, either through direct interaction of analyte with the matrix, which is present in larger quantities, or by changes in surface phenomena at the more dilute concentrations. In fact, the one-electron oxidation and reduction processes as well as the two-electron reduction process occur to a lesser extent as the concentration is increased. The greatest concentration effect is observed for one-electron reduction, which increases more than thirtyfold when the concentration changes from 0.05 to $0.005 \mathrm{M}$.

Once the Simbroc program was validated, it was applied to various chemical systems. The results shown in Table 3 are those obtained for an acetal, which undergoes extensive two-electron oxidation; the peptide DDAVP-ACOH, which undergoes extensive twoelectron reduction; methylene blue, which undergoes both one- and two-electron reductions; and pentaphenylalanine, which undergoes combined processes. The results presented in Table 3 indicate that Simbroc has no problem evaluating major processes while keeping the background level at values that compare well with those observed in the mass spectra. Once the task of developing a method of reliably isolating and evaluating the background level and redox extent is achieved, it becomes possible to study the effects of experimental parameters on the "true" extent of reduction and oxidation and on the background signal which itself can vary with experimental conditions.

An experimental FAB source parameter that was reported to affect the "apparent" reduction given by $(A+2) / A$ ratios alone was the primary beam energy [15]. Experiments conducted in our laboratory using a $\mathrm{Cs}^{+}$ion gun, however, did not show an important

Table 3. Background levels and extent of reduction/oxidation processes determined by Simbroc for various chemical systems measured at flux $0.018 \mu \mathrm{A} / \mathrm{mm}^{2}$

\begin{tabular}{|c|c|c|c|c|c|c|c|}
\hline $\begin{array}{l}\text { Compound } \\
\text { (matrix) }\end{array}$ & Back $_{1}$ & Back $_{2}$ & $\begin{array}{l}\mathrm{Ox}_{2} \\
(\%)\end{array}$ & $\begin{array}{l}O x_{1} \\
(\%)\end{array}$ & $\begin{array}{c}\operatorname{Red}_{0} \\
(\%)\end{array}$ & $\begin{array}{c}\operatorname{Red}_{1} \\
(\%)\end{array}$ & $\begin{array}{c}\operatorname{Red}_{2} \\
(\%)\end{array}$ \\
\hline $\begin{array}{l}\text { Acetal } \\
\text { (neat) }\end{array}$ & 0.03 & 0.01 & 90.1 & 2.6 & 7.1 & 0.21 & 0.05 \\
\hline $\begin{array}{l}\text { DDAVP-AcOH } \\
\text { (thioglyceroll }^{\mathbf{b}}\end{array}$ & 1.8 & 0.0 & 2.1 & 1.9 & 22.1 & 1.9 & 71.9 \\
\hline $\begin{array}{l}\text { Methylene blue } \\
\text { (glycerol) }\end{array}$ & 1.0 & 2.2 & 1.5 & 1.0 & 46.8 & 41.0 & 9.8 \\
\hline $\begin{array}{l}\text { FFFFF-OH } \\
\text { (glycerol) }^{c}\end{array}$ & 3.9 & 3.2 & 4.0 & 4.2 & 86.4 & 2.4 & 3.0 \\
\hline
\end{tabular}

\footnotetext{
4-Decyl-2-phenyl-1,3-dioxolane.

Deaminoarginine vasopressin (disulfide bridge-containing pep-

'Pentaphenylalanine.
} tide). 
energy dependence when $(A+2) / A$ ratios were measured for various peptide samples [28]. Because the isolopic peak measurements alone can sometimes be deceiving, it was interesting to determine whether the primary beam energy affects the redox extent or background level. The Simbroc results for pressinoic acid in glycerol $(0.025 \mathrm{M})$ analyzed at a primary beam density of $0.018 \mu \mathrm{A} / \mathrm{mm}^{2}$ are presented in Table 4 . The background level for pressinoic acid in glycerol is very low, thereby causing a somewhat large relative error in the background values. Nevertheless, no significant effect on background can be observed at the three different primary beam energies studied. Comparison of the redox values shows only slightly elevated values of one-electron oxidation and one- and two-electron reduction at the lowest beam energy of $8 \mathrm{keV}$. The background and redox values obtained at 12 and 16 keV, however, show no primary beam energy effect at all. Because most experiments conducted in this study were performed at a beam energy of $14 \mathrm{keV}$, this parameter does not play an important role in the following discussion.

The effects of concentration and beam density on the true redox extent values were studied for bradykinin and pentaphenylalanine samples in glycerol. The peak intensities measured in the molecular ion region of interest $\left(I_{-3}-I_{4}\right)$ for a bradykinin sample under various experimental conditions are shown in Table 5. For this sample, the base peak in the mass region studied occurs at the parent ion mass $\left(I_{0}\right)$ for all conditions used in this study. A similar set of raw data were obtained for pentaphenylalanine in glycerol. These sets of observed peak intensities were then entered into the Simbroc program to determine the "true" levels of reduction, oxidation, and background signal. The extent of the different redox processes and the background level as determined by Simbroc for the bradykinin and pentaphenylalanine results are shown
Table 4. Effect of primary beam energy on background level and redox processes for a pressinoic acid sample in glycerol $\left(0.25 \mathrm{M}, 0.018 \mu \mathrm{A} / \mathrm{mm}^{2}\right)$

\begin{tabular}{cccccccc}
\hline $\begin{array}{c}\text { Primary } \\
\text { beam energy } \\
\text { (keV) }\end{array}$ & Back, $_{1}$ & Back, $_{2}$ & O. $x_{2}$ & Ox, & Red $_{0}$ & Red $_{1}$ & Red $_{2}$ \\
\hline 8 & 1.31 & 0.00 & 0.57 & 3.6 & 85.3 & 4.3 & 6.3 \\
12 & 0.00 & 0.00 & 1.1 & 2.6 & 87.2 & 3.3 & 5.8 \\
16 & 0.00 & 0.00 & 1.0 & 2.7 & 86.8 & 3.6 & 5.9 \\
\hline
\end{tabular}

in Tables 6 and 7, respectively. Also shown in Tables 6 and 7 are the "apparent" reduction values that are calculated by subtracting the theoretical second isotopic peak intensities from the measured $A+2$ peak intensities. These apparent values can be compared directly with the $\operatorname{Red}_{2}$ values provided by the computer program.

In the bradykinin set of results shown in Table 6, the background levels calculated by the Simbroc program are somewhat lower than the $I_{-3}$ values and experimentally observed background peaks would indicate. The absolute error associated with these background values is on the order of $1 \%$, which, when distributed over all of the other variables involved in the calculations, results in very small relative errors in the redox extent values. For backgromind levels lower than approximately $3 \%$, the relative error in the background values calculated by Simbroc may appear to be substantial. In absolute terms, however, an error of $1 \%$ is not important. Furthermore, as the background level increases, the relative error decreases significantly. The background levels appear to be higher at the lowest concentration of $0.005 \mathrm{M}$ and are affected to a lesser extent by changes in concentration at the higher concentrations. Bccause Back 1 and Back 2 values do not generally vary by more than $10 \%$ relatively, the results indicate that the background is distributed evenly in the molecular ion cluster region (constant pattern, Fig-

Table 5. Peak intensities measured in the molecular ion region for a bradykinin sample in glycerol

\begin{tabular}{|c|c|c|c|c|c|c|c|c|c|}
\hline \multicolumn{2}{|c|}{ Experimental conditions } & \multirow{2}{*}{\multicolumn{8}{|c|}{ Relative peak intensities (\%) }} \\
\hline \multirow{2}{*}{$\begin{array}{c}\text { Beam } \\
\text { Density } \\
\left(\mu \mathrm{A} / \mathrm{mm}^{2}\right)\end{array}$} & \multirow{2}{*}{$\begin{array}{l}\text { Concentration } \\
\text { in glycerol } \\
\text { (M) }\end{array}$} & & & & & & & & \\
\hline & & $I_{3}$ & $I_{-2}$ & $\mathrm{I}_{-1}$ & $\mathrm{I}_{0}$ & $I_{1}$ & $t_{2}$ & $I_{3}$ & $\mathrm{I}_{4}$ \\
\hline \multirow[t]{4}{*}{0.018} & 0.005 & 2.33 & 7.41 & 13.82 & 100.00 & 70.97 & 33.06 & 11.95 & 4.02 \\
\hline & 0.02 & 2.16 & 8.10 & 10.08 & 100.00 & 63.08 & 26.71 & 8.95 & 2.94 \\
\hline & 0.04 & 1.83 & 7.90 & 8.34 & 100.00 & 62.80 & 24.99 & 7.99 & 2.50 \\
\hline & 0.08 & 1.32 & 6.96 & 6.49 & 100.00 & 61.03 & 23.56 & 7.16 & 2.10 \\
\hline \multirow[t]{4}{*}{0.032} & 0.005 & 3.45 & 8.87 & 15.03 & 100.00 & 71.11 & 33.34 & 12.39 & 4.56 \\
\hline & 0.02 & 2.96 & 9.34 & 11.21 & 100.00 & 63.26 & 27.45 & 9.60 & 3.46 \\
\hline & 0.04 & 2.60 & 9.16 & 9.53 & 100.00 & 61.90 & 25.91 & 8.72 & 3.16 \\
\hline & 0.08 & 2.14 & 8.57 & 8.35 & 100.00 & 62.10 & 25.01 & 8.14 & 2.63 \\
\hline \multirow[t]{4}{*}{0.053} & 0.005 & 3.66 & 9.17 & 15.19 & 100.00 & 70.49 & 33.13 & 12.36 & 4.63 \\
\hline & 0.02 & 1.93 & 7.80 & 10.99 & 100.00 & 64.13 & 27.72 & 9.41 & 3.03 \\
\hline & 0.04 & 2.20 & 8.16 & 10.36 & 100.00 & 63.13 & 26.74 & 8.96 & 2.95 \\
\hline & 0.08 & 1.97 & 8.02 & 7.79 & 100.00 & 62.39 & 24.81 & 8.01 & 2.57 \\
\hline
\end{tabular}


Table 6. Extent of reduction/oxidation process obtained using Simbroc and extent of "apparent" reduction measured for bradykinin sample in glycerol

\begin{tabular}{|c|c|c|c|c|c|c|c|c|c|}
\hline \multicolumn{2}{|c|}{ Experimental conditions } & \multirow{2}{*}{\multicolumn{2}{|c|}{$\begin{array}{l}\text { Background } \\
\text { level (\%) }\end{array}$}} & \multirow{2}{*}{\multicolumn{5}{|c|}{ Relative peak intensities (\%) }} & \multirow[b]{3}{*}{$\operatorname{Red}_{\mathrm{app}}$} \\
\hline \multirow{2}{*}{$\begin{array}{c}\text { Beam } \\
\text { density } \\
\left(\mu \mathrm{A} / \mathrm{mm}^{2}\right)\end{array}$} & \multirow{2}{*}{$\begin{array}{c}\text { Concentration } \\
\text { in glycerol } \\
\text { (M) }\end{array}$} & & & & & & & & \\
\hline & & Back $_{1}$ & Back $_{2}$ & $\overline{\mathrm{Ox}_{2}}$ & $O x_{1}$ & $\operatorname{Red}_{0}$ & Red $_{1}$ & $\overline{\operatorname{Red}_{2}}$ & \\
\hline \multirow[t]{4}{*}{0.018} & 0.005 & 1.4 & 1.6 & 4.9 & 7.0 & 75.8 & 7.9 & 4.4 & 11.5 \\
\hline & 0.02 & 1.0 & 1.0 & 6.4 & 4.1 & 84.6 & 1.2 & 3.7 & 5.2 \\
\hline & 0.04 & 0.9 & 1.0 & 6.4 & 2.7 & 87.7 & 0.7 & 2.5 & 3.4 \\
\hline & 0.08 & 1.1 & 0.8 & 5.5 & 2.0 & 90.9 & 0.0 & 1.6 & 2.0 \\
\hline \multirow[t]{4}{*}{0.032} & 0.005 & 2.0 & 2.2 & 5.7 & 7.0 & 75.2 & 7.9 & 4.3 & 11.8 \\
\hline & 0.02 & 1.5 & 1.4 & 7.0 & 4.3 & 83.4 & 1.3 & 4.0 & 5.9 \\
\hline & 0.04 & 1.4 & 1.3 & 7.1 & 3.1 & 86.3 & 0.0 & 3.5 & 4.4 \\
\hline & 0.08 & 1.0 & 1.0 & 7.0 & 2.3 & 87.8 & 0.0 & 2.9 & 3.5 \\
\hline \multirow[t]{4}{*}{0.053} & 0.005 & 2.1 & 2.2 & 5.9 & 7.0 & 75.3 & 7.4 & 4.4 & 11.6 \\
\hline & 0.02 & 1.0 & 1.0 & 6.0 & 5.0 & 82.7 & 2.3 & 1.0 & 6.2 \\
\hline & 0.04 & 1.0 & 1.0 & 6.4 & 4.3 & 84.3 & 1.3 & 3.7 & 5.2 \\
\hline & 0.08 & 1.0 & 1.1 & 6.5 & 2.1 & 88.6 & 0.1 & 2.7 & 3.3 \\
\hline
\end{tabular}

ure 2a). The two-electron oxidation process appears to be independent of concentration at concentrations greater than $0.02 \mathrm{M}$. It is somewhat lower at the lowest concentration of $0.005 \mathrm{M}$. An increase in beam density appears to cause an increase in the extent of the twoelectron oxidation process at this low concentration but does not affect the process in solutions of higher concentration. A more noticeable trend in the extent of redox processes with concentration is observed for the onc-elcctron oxidation and for the one and two-electron reduction processes. In each case, the processes occur to a greater extent as the concentration is lowered. The one-electron reduction process appears to be the most vulnerable to changes in concentration because it drops dramatically over the concentration range studied. Increases in beam density do not appear to affect the extent of these redox processes to a large extent under the experimental conditions used. The extent of intact parent ion signal, $\operatorname{Red}_{0}$, increases with increasing concentration, thereby suggesting that the analyte is more likely to undergo reduction/oxidation reactions as the sample becomes more diluted. This observation would imply that the matrix assists in the reduction / oxidation of the analyte for this system. It is not surprising to note that the intact parent ion signal decreases with increasing beam density because it is logical to expect more damage to the parent ion at a higher bombarding beam density. Comparison of the extent of two-electron reduction $\left(\operatorname{Red}_{2}\right)$ with the apparent reduction $\left(\operatorname{Red}_{\mathrm{app}}\right.$ ) indicated that the "true"

Table 7. Extent of reduction/oxidation process obtained using Simbroc and extent of "apparent" reduction measured for pentaphenylalanine sample in glycerol

\begin{tabular}{|c|c|c|c|c|c|c|c|c|c|}
\hline \multicolumn{2}{|c|}{ Experimental conditions } & \multirow{2}{*}{\multicolumn{2}{|c|}{$\begin{array}{l}\text { Background } \\
\text { level }(\%)\end{array}$}} & \multirow{2}{*}{\multicolumn{5}{|c|}{ Extent of Redox Processes (\%) }} & \multirow[b]{3}{*}{$\operatorname{Red}_{\text {app }}$} \\
\hline \multirow{2}{*}{$\begin{array}{c}\text { Beam } \\
\text { density } \\
\left(\mu \mathrm{A} / \mathrm{mm}^{2}\right)\end{array}$} & \multirow{2}{*}{$\begin{array}{l}\text { Concentration } \\
\text { in glycerol } \\
\text { (M) }\end{array}$} & & & & & & & & \\
\hline & & Back $_{1}$ & Back $_{2}$ & $\mathrm{Ox}_{2}$ & $O x_{1}$ & $\operatorname{Red}_{0}$ & $\operatorname{Red}_{1}$ & $\operatorname{Red}_{2}$ & \\
\hline \multirow[t]{4}{*}{0.018} & 0.005 & 7.4 & 7.3 & 3.3 & 4.7 & 84.6 & 4.9 & 2.5 & 11.4 \\
\hline & 0.02 & 3.9 & 3.2 & 4.0 & 4.2 & 86.4 & 2.4 & 3.0 & 7.7 \\
\hline & 0.04 & 3.8 & 3.1 & 3.0 & 3.8 & 87.1 & 2.9 & 3.1 & 8.0 \\
\hline & 0.08 & 3.4 & 3.0 & 2.6 & 3.0 & 89.9 & 2.7 & 1.8 & 6.1 \\
\hline \multirow[t]{4}{*}{0.032} & 0.005 & 9.5 & 10.9 & 4.2 & 6.9 & 79.3 & 5.9 & 3.8 & 15.1 \\
\hline & 0.02 & 5.2 & 5.0 & 4.1 & 4.8 & 84.1 & 4.3 & 2.7 & 9.5 \\
\hline & 0.04 & 4.5 & 4.2 & 3.4 & 3.9 & 86.0 & 2.6 & 4.0 & 9.4 \\
\hline & 0.08 & 4.5 & 3.7 & 3.3 & 3.4 & 87.9 & 2.6 & 2.7 & 7.9 \\
\hline \multirow[t]{4}{*}{0.053} & 0.005 & 8.2 & 9.3 & 4.0 & 6.5 & 80.4 & 6.1 & 3.0 & 13.3 \\
\hline & 0.02 & 9.0 & 9.1 & 4.9 & 6.2 & 81.1 & 4.4 & 3.4 & 13.4 \\
\hline & 0.04 & 10.0 & 10.0 & 4.3 & 4.9 & 83.5 & 3.9 & 3.6 & 14.1 \\
\hline & 0.08 & 8.9 & 8.7 & 4.7 & 4.5 & 85.0 & 2.8 & 3.1 & 12.0 \\
\hline
\end{tabular}


extent of reduction occurs to a much lesser extent than would be expected by the apparent values. The discrepancy is particularly important at the lower concentrations. Furthermore, the concentration effect is not as important in two-electron reduction processes, ranging by approximately two times the lowest value $(0.08 \mathrm{M})$, whereas the apparent values differ by approximately four to five times over the concentration range studied. The flux effect on the two-electron reduction process in either case appears to be most important at the higher concentrations of 0.04 and $0.08 \mathrm{M}$, where the oneelectron reduction process occurs to a much lesser extent.

The results obtained using Simbroc for the data set of pentaphenylalanine in glycerol are shown in Table 7. It should be noted that the experimental data obtained at $0.053 \mu \mathrm{A} / \mathrm{mm}^{2}$ has a larger error associated with it (approximately 30\% relative) because this sample undergoes more sputtering at this higher beam density, thereby causing more instability in the source during the analysis. The background values found in Table 7 at fluxes of 0.018 and $0.032 \mu \mathrm{A} / \mathrm{mm}^{2}$ demonstrate that the background signal for this chemical system decreases with increasing concentration. As the beam density is increased, the background levels tend to increase at each concentration used in this study. The molecular ion regions for a $0.02 \mathrm{M}$ solution of pentaphenylalanine in glycerol at primary beam fluxes of $0.018,0.032$, and $0.053 \mu \mathrm{A} / \mathrm{mm}^{2}$ are shown in Figure $4 a-c$, respectively. These profiles illustrate that the background signal for this system does incleed increase with increasing primary beam density. Figure 4 also confirms the agreement between the magnitude of background values provided by Simbroc and those observed experimentally. It is interesting to note that the experimental background observed in Figure 4a has a "picket-fence" pattern that disappears as the primary beam flux is increased. This trend is picked up by Simbroc, which calculates a "picket-fence" profile with Back 1 and Back 2 values of 3.9 and 3.2 and 5.2 and 5.0 at flux values of 0.018 and $0.032 \mu \mathrm{A} / \mathrm{mm}^{2}$, respectively, whereas it calculates a constant background of 9.0 and 9.1 at the higher flux of 0.053 $\mu \mathrm{A} / \mathbf{U n}^{2}$.

The two-electron oxidation process in this case does not show any clear trends with concentration, whereas the one-electron processes $\left(\mathrm{Ox}_{1}\right.$ and $\left.\mathrm{Red}_{1}\right)$ both show a decrease in the extent of redox processes with increasing concentration. These latter processes also generally appear to occur to a greater extent as the primary beam density is increased. The percentage of intact parent ion, as in the case of the bradykinin data set, increases with increasing concentration. Contrary to the large concentration effect suggested by the apparent reduction values, Red $_{\text {app }}$, the true two-electron reduction process, $\operatorname{Red}_{2}$, does not show any clear trend. In fact, the true two-electron reduction process is practically independent of either concentration or primary beam density, whereas the apparent reduction process
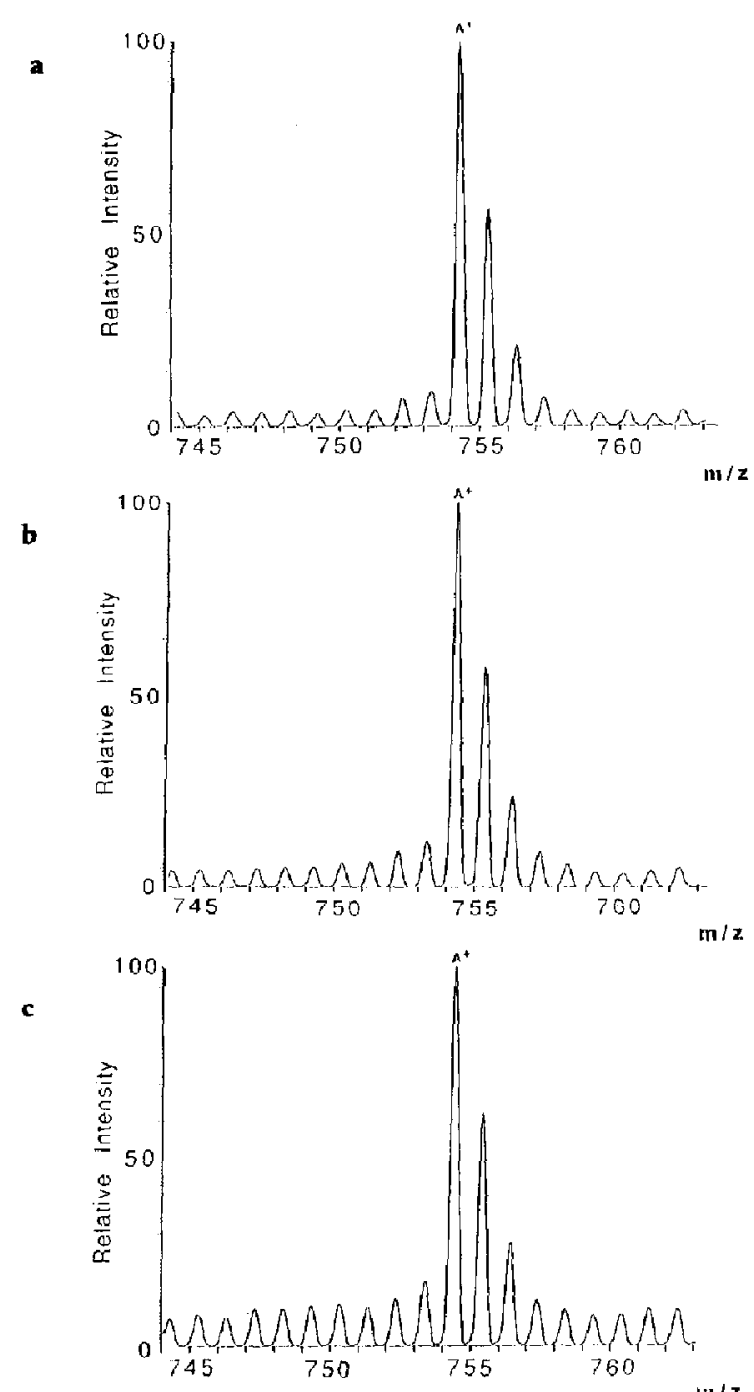

Figure 4. Molecular ion cluster protiles of pentaphenylalanine in glycerol observed at primary beam densities of (a) 0.018 , (b) 0.032 , and (c) $0.053 \mu \mathrm{A} / \mathrm{mm}^{2}$; concentration $0.02 \mathrm{M}$; primary heam energy $14 \mathrm{keV}$.

shows a substantial decrease in reduction with increasing concentration at 0.018 and $0.032 \mu \mathrm{A} / \mathrm{mum}^{2}$ and a significant increase in reduction when the beam density is increased. Furthermore, besides the conflicting trends, the true reduction extent values are approximately three to five times smaller than those suggested from the apparent values. It is the background levels as shown by Back 1 and Back values, not Red $_{2}$ values, therefore, that follow the trends present in the apparent reduction data. It is very important, therefore, to be able to decipher the background signal from the overall peak intensities measured in the molecular ion cluster region so that misleading conclusions are not drawn from experimental data. 


\section{Conclusion}

Although often omitted in the literature, it is important to report all pertinent experimental parameters when indicating the extent of reduction/oxidation observed in a chemical system. The parameters that are most influential in the variation of peak intensities are the analyte concentration, the primary beam density, and the matrix compusition. The experimental conditions affect not only the extent of reduction/oxidation processes but also the levels of background signal due to chemical damage during FAB/LSIMS analysis. The mathematical model on which Simbroc is based can be used successfully to deconvolute the experimental peak intensities in the molecular ion cluster region. Once properly isolated, the effect of experimental parameters on the background level and on the extent of "true" rather than "apparent" redox processes can be studied. This method is particularly important in cases in which background is significant, thereby creating false trends. Thus, the approach described here allows the evaluation of the many species generated under FAB/LSIMS conditions and is able to provide true trends that are essential in the establishment of the actual mechanisms involved in these redox processes.

\section{Acknowledgment}

The authors acknowledge the financial support of the Natural Science and Engineering Rescarch Council of Canada that has permitted this study.

\section{References}

1. Pelzer, G.; De Pauw, E.; Dung, D. V.; Marien, J. J. Pliys. Chem. 1984, 88, 5065.

2. Cerny, R. L.; Gross, M. L. Anal. Chem. 1985, 57, 1160.

3. Buko, A. M.; Fraser, B. A. Biomed. Mass Spectrom. 1985, 12 , 577.

4. Fujita, Y.; Matsuo, T.; Sakurai, T.; Matsuda, H.; Katakuse, 1. Int. I. Mass Spectrom. Ton Processes 1985, 63, 231

5. Gale, P. J.; Bentz, B. L.; Chait, B. T.; Field, F. H.; Cotter, R. J. Anal. Chem. 1986, 58, 1070 .

6. Musselman, B. D.; Watson, J. T. Bioned. Environ. Mass Spectrom. 1987, 14. 247

7. Kazakoff, C. W.; Rye, R. T. B.; Tee, O. S. Can. J. Chen. 1987, $65,718$.

8. Wirth, K. P.; Junker, E.; Rollgen, F. W.; Fonrobert, P.; PrzybyIski, M. J. Chem. Soc. Chem. Commun. 1987, 1387.

9. Kyranos, J. N.; du Sorbier, B. M.; Wronka, J.; Vouros, P.; Kirby, D. P. Org. Mass Spectrom. 1988, 23, 443.

10. Burinsky, D. J.; Dilliplane, R. L.; Di Donato, G. C.; Busch, K L. Org. Mass Spectrom. 1988, 23, 231.
11. Hand, O. W.; Detter, L. D.; Cooks, R. G.; Walton, R. A. Mass Spectrom. Ret. 1988, 7. 465.

12. Brown, S. M.; Busch, K. L. Rapid Conmun. Mass Spectrom. $1988,2,256$.

13. Visentini, J.; Thibault, P.; Bertrand, M. J. Proceedings of the 37 th ASMS Conference on Mass Spectrometry and Allied Topics; Miami, FL, 1989; p 887.

14. Visentini, I.; Gauthier, J.; Bertrand, M. I. Rapid Commun. Mass Spectrom. 1989, 3, 390.

15. Kazakoff, C.; Rye, R. T. B.; Tee, O. S. Can. I. Chem. 1989, 67, 183.

16. Santana-Marques, M. G. O.; Ferrer-Correia, A. J. V.; Gross, M. L. Anal. Chem. 1989, 61, 1442.

17. Thibault, P.; Quilliam, M. A.; Jamieson, W. D.; Boyd, R. K. Biomed. Environ. Mass Spectrom, 1989, 18, 373.

18. Laramee. J. A.; Arbugast, B.; Deinzer, M. L. Anai. Chem, 1989, $61,2154$.

19. Kyranos, J. N.; Vouros, P. Biomed. Enoiron. Mass Spectrom. $1990,19,628$

20. Reynolds, J. D.; Cook, K. D. J. Am. Soc. Mass Spectrom. 1990, $1,149$.

21. Vekey, K, Int. J. Mass Spectrom. Ion Processes 1990, 97, 265.

22. Busch, K. L. Cherntracts $1990,2,449$.

23. Agnello, A.; De Pauw, E. Org. Mass Spectrom. 1991, $26,175$.

24. Nedderman, A. N. R.; Williams, D. H. Biol. Mass Spectrom. $1991,20,289$.

25. Visentini, J.; Nguyen, P. M.: Bertrand, M. J. Rapid Commun. Mass Spectron. 1991, 5, 586.

26. Kazakoff, C. W.; Rye, R. T. B. Org. Mass Spectrom. 1991, 26, 154.

27. Vekey, K.: Zerilli, L. F. Org. Mass Spectrom. 1991, 26, 939.

28. Bertrand, M. J.; Visentini, J.; Paul, G. J. C.; Zidarov, D. Rapid Commun. Mass Spectrom. 1992, 6, 485, and references therein.

29. Visentini, J.; Bertrand, M. J., unpublished results

30. Allard, M.; Visentini, J.; Bertrand, M. J. Proceedings of the 40th ASMS Conference on Mass Spectrometry and Allied Topics: Washington, D.C., May 31-June 5, 1992; p 1409.

31. Ishikawa, K.; Niwa, Y. Biomed. Environ. Mass Spectrom. 1986, $13,373$.

32. Bertrand, M. J.; Thibault, P.; Evans, M. J.; Zidarov, D. Biomed. Ermiron. Mass Spectrom. 1987, 14, 249.

33. Zidarov, D.; Thibault, P.; Evans, M. J.; Bertrand, M. J. Biomed. Enoiron. Mass Spectroin. 1990, 19, 13.

34. Zidarov, D.; Faubert, D.; Visentini, J.; Bertrand, M. J. Proceedings of the 39th ASMS Conference on Mass Spectrometry and Allied Topics; Nashville, TN, May 19-24, 1991; p 1123.

35. Baldwin, M. A.; Welham, K. J.; Toth, I.; Gibbons, W. A. Org. Mass Spectrom. 1988, 23, 697.

36. Zha, Q.; Bailey, M. D.; Bertrand, M. J. Can. J. Appl. Spectros. 1990, 35, 141.

37. Paul, G. J. C.; Bourg, 5; Bertrand, M. J. Rapid Commun. Mass Spectron. 1992, 6, 85.

38. Paul, G. J. C.; Bourg, S.; Bertrand, M. J. J. Ant. Soc. Mass Spectrom. in press.

39. Blom, K. F. Org. Mass. Spectrom. 1988, 23, 194.

40. Rosenbrock, H. H. Comput. J. 1960, 3, 175. 